Applied Mathematical Sciences, Vol. 1, 2007, no. 15, 739 - 747

\title{
The Fixed Point of Fuzzy Complex Number-Valued Mapping
}

\author{
Shenquan Ma ${ }^{1}$ and Dejun Peng \\ Department of Mathematics, Hainan Normal University \\ Hainan, Haikou, 571158, P.R. of China
}

\begin{abstract}
In this paper, we introduce the concepts of fuzzy complex number, the operations of fuzzy complex number, the concepts of convergence for fuzzy complex number-valued sequence and the fuzzy complex numbervalued mapping. Then the fixed point of fuzzy complex number-valued mapping is discussed, some existent theorems of this mapping are given. It will establish a foundation for researching fuzzy complex analysis .
\end{abstract}

Keywords: Fuzzy complex set, Fuzzy complex number, Fuzzy complex number-valued mapping, The fixed point of fuzzy complex number-valued mapping.

\section{Introduction}

Since the concept of fuzzy complex numbers was first introduced by J.J.Buckley in 1989, many papers were devoted to studying the problems of the concept of fuzzy complex numbers, operations, mappings between fuzzy complex number sets, and problems of the limit, continuity, differential and integral of fuzzy complex number-valued mappings were further researched from different aspects, and many important results were gained(see [1]-[11]). The frame of fuzzy complex analysis theory has had its primary shape and tends to form a new branch of mathematics step by step (see [6]). This new branch subject will be widely applied in fuzzy system theory, especially in fuzzy dynamical system theory, and will also be widely applied in the field of computational intelligence (see [6][11]). It is well known that the fixed point theory plays a very important role in mathematic theory and applications, in particular it has important value in finding root of algebraic equation and numerical mathematics. However few papers were devoted to researching the

\footnotetext{
${ }^{1}$ Corresponding author. e-mail: msq6202@hainnu.edu.cn
} 
problem of the fixed point of fuzzy complex number-valued mapping. Therefore, based on author's researches in recent years, this problem is investigated in this paper and gain some important conclusions.

\section{$2 \quad$ Preliminary concepts}

Let $R$ be a set of real number, $C=\{x+i y \mid x \in R, y \in R, i=\sqrt{-1}\}$ a field of complex number, a finite closed interval $X=\left[X^{-}, X^{+}\right]$is called a closed interval number on $R, I(R)$ denotes the set of all closed interval numbers on $R$.

For arbitrary interval $X=\left[X^{-}, X^{+}\right], Y=\left[Y^{-}, Y^{+}\right] \in R, Z=X+i Y=$ $\{x+i y \in C \mid x \in X, y \in Y, i=\sqrt{-1}\}$ is called a closed complex interval number, $I(C)=\{Z=X+i Y \mid X, Y \in I(R), i=\sqrt{-1}\}$ denotes the set of all closed complex interval numbers on $C,{ }^{\prime} *=\{+,-, \times, \div\}^{\prime}$ is a binary operation on $I(C)$,(when $\left.*=Z_{1} \div Z_{2}, 0 \notin \operatorname{supp} Z_{2}\right)$

Definition: For $\forall Z_{k}=X_{k}+i Y_{k}=\left[X_{k}^{-}, X_{k}^{+}\right]+i\left[Y_{k}^{-}, Y_{k}^{+}\right] \in I(C),(k=1,2)$, $Z_{1} * Z_{2}=\left\{z \mid \exists\left(z_{1}, z_{2}\right) \in Z_{1} \times Z_{2}, z=z_{1} * z_{2}\right\}$ and $X=\left[X^{-}, X^{+}\right], Y=$ $\left[Y^{-}, Y^{+}\right] \in I(R)$. Specifically, for $k \in R^{+}=[0,+\infty)$, let $k \cdot X=\left[k X^{-}, k X^{+}\right]$, Let $k \cdot Z=(k X)+i(k Y)=\left[k X^{-}, k X^{+}\right]+i\left[k Y^{-}, k Y^{+}\right]$, Let $Z_{k}=X_{k}+i Y_{k}(k=$ $1,2) \in I(C), Z_{1} \subseteq Z_{2} \Leftrightarrow X_{1} \subseteq X_{2}, Y_{1} \subseteq Y_{2}$. If $X, Y$ are convex sets on $R$, $Z \subseteq C$ and

$$
Z=\{x+i y \in C \mid x \in X, y \in Y\}
$$

then $Z$ is a convex set on $C$.

Let $Z \subseteq C, Z$ is called a convex set on $C$, if and only if $z_{n}=x_{n}+i y_{n} \in$ $Z,(n=1,2, \cdots)$, and

$$
\lim _{x \rightarrow \infty} z_{n}=\lim _{x \rightarrow \infty}\left(x_{n}+i y_{n}\right)=z=x+i y \Rightarrow^{\prime} z=x+i y \in C^{\prime}
$$

Complex set $Z$ is called a closed convex complex set, if it satisfies (1) and (2). Clearly, we have:

Theorem 2.1 Let $C$ be a field of complex number, $Z \subseteq C$, and $Z$ be a bounded set, then $Z$ is called a closed convex set, if and only if $Z$ be a closed complex interval number.

\section{The concept and operation of fuzzy complex sets and fuzzy complex numbers}

Definition 3.1 Let $C$ be a field of complex number, mapping $\tilde{Z}: C \rightarrow[0,1]$ is called a fuzzy complex set, $\tilde{Z}(z)$ is called the membership function of fuzzy set $\tilde{Z}$ for $z, F(C)=\{\tilde{Z} \mid \tilde{Z}: C \rightarrow[0,1]\}$ denotes all fuzzy complex sets on $\mathrm{C}$. 


\section{Definition 3.2}

(1) $\tilde{Z}_{\alpha}=\{z=x+i y \in C \mid \tilde{Z}(z)=\tilde{Z}(x+i y) \geq \alpha\}$ is called $\alpha$-cut set of $\tilde{Z}$.

(2) $\tilde{Z}_{\alpha}=\{z=x+i y \in C \mid \tilde{Z}(z)=\tilde{Z}(x+i y)>\alpha\}$ is called strong $\alpha$-cut set of $\tilde{Z}$.

(3) $\tilde{Z}_{0}=\operatorname{supp} \tilde{Z}=\{z=x+i y \in C \mid \tilde{Z}(z)=\tilde{Z}(x+i y)>0\}$ is called support set of $\tilde{Z}$.

\section{Definition 3.3}

(i) $\tilde{Z} \in F(C)$ is called a convex fuzzy complex set on $C$, if and only if for $\forall \alpha \in[0,1], \tilde{Z}_{\alpha}$ is a convex complex set on $C$.

(ii) $\tilde{Z} \in F(\underset{\tilde{Z}}{C})$ is called a closed fuzzy complex set on $C$, if and only if for $\forall \alpha \in[0,1], \tilde{Z}_{\alpha}$ is a closed complex set on $C$.

(iii) If $\tilde{Z} \in F(C)$, supp $\tilde{Z}$ is a bounded set, or for $\forall \alpha \in[0,1], \tilde{Z}_{\alpha}$ is a bounded set, then $\tilde{Z}$ is called a closed convex fuzzy complex set, if and only if for $\forall \alpha \in[0,1], \tilde{Z}_{\alpha}$ is a closed complex interval number (ordain $\emptyset$ is a closed complex interval number on $C$ ).

\section{Definition 3.4}

(i) $\tilde{Z} \in F(C)$ is a normal fuzzy complex set on $C$, if and only if $\{z \in C \mid \tilde{Z}(z)=$ 1\} $\neq \emptyset$;

(ii) if supp $\tilde{Z}$ is abounded complex set, $\tilde{Z}$ is called a finite fuzzy complex set; (iii) if for $\forall \alpha \in(0,1], \tilde{Z}_{\alpha}$ is a bounded complex set, $\tilde{Z}$ is called a bounded fuzzy complex set.

The finite fuzzy complex set and the bounded fuzzy complex set are generally called bounded fuzzy complex set.

Definition 3.5 A normal convex fuzzy complex set on $C$ is called a fuzzy complex number; a normal closed convex fuzzy complex set is called a closed fuzzy complex number ; a normal bounded closed convex fuzzy complex set is a bounded closed fuzzy complex number. we write

$$
F_{0}(C)=\{\tilde{Z} \mid \tilde{Z} \text { be a bounded closed fuzzy complex number on } C\}
$$

As a whole bounded closed fuzzy complex number on $C, F_{0}(C)$ is a very important set, and it plays a very significant role in the research of fuzzy complex analysis. From the conceptual analysis above, it is obvious that bounded closed fuzzy complex number possesses nicer property, its operation becomes very facility and it provides much advantage to us in application. Since its level complex set is closed complex interval numbers. In the following we present the operation of fuzzy complex number.

Let $*$ be an algebraic operation on $C$;

$$
*: C \times C \rightarrow C, \quad\left(z_{1}, z_{2}\right) \rightarrow w=z_{1} * z_{2}
$$

Since the extension principle of complex fuzzy sets, we have operations 
among fuzzy numbers as follows:

$$
\begin{gathered}
*: F_{0}(C) \times F_{0}(C) \rightarrow F_{0}(C) \\
(\tilde{E}, \tilde{D}) \rightarrow \tilde{E} * \tilde{D}=\bigcup_{\alpha \in(0,1]} \alpha\left(E_{\alpha} * D_{\alpha}\right)
\end{gathered}
$$

where

$$
\begin{gathered}
E_{\alpha} * D_{\alpha}=\left\{w \mid \exists\left(z_{1}, z_{2}\right) \in E_{\alpha} \times D_{\alpha}, z_{1} * z_{2}=w\right\} \\
(\tilde{E} * \tilde{D})(w)=\bigvee_{z_{1} * z_{2}=w}\left(\tilde{E}\left(z_{1}\right) \wedge \tilde{D}\left(z_{2}\right)\right)
\end{gathered}
$$

For arithmetic operation ",,$+- \times, \div "$ we have

$$
\begin{aligned}
(\tilde{E}+\tilde{D})(w) & =\underset{z_{1}+z_{2}=w}{\vee}\left(\tilde{E}\left(z_{1}\right) \wedge \tilde{D}\left(z_{2}\right)\right) \\
& =\vee_{z_{1} \in C}\left(\tilde{E}\left(z_{1}\right) \wedge \tilde{D}\left(w-z_{1}\right)\right) \\
(\tilde{E}-\tilde{D})(w) & =\underset{z_{1}-z_{2}=w}{\vee}\left(\tilde{E}\left(z_{1}\right) \wedge \tilde{D}\left(z_{2}\right)\right) \\
& =\vee_{z_{1} \in C}\left(\tilde{E}\left(z_{1}\right) \wedge \tilde{D}\left(z_{1}-w\right)\right) \\
(\tilde{E} \cdot \tilde{D})(w) & =\vee_{z_{1} \cdot z_{2}=w}^{\vee}\left(\tilde{E}\left(z_{1}\right) \wedge \tilde{D}\left(z_{2}\right)\right) \\
(\tilde{E} \div \tilde{D})(w) & =\underset{z_{1} \div z_{2}=w}{\vee}\left(\tilde{E}\left(z_{1}\right) \wedge \tilde{D}\left(z_{2}\right)\right) \quad(0 \notin \text { supp } \tilde{D})
\end{aligned}
$$

According to the above definition, we have operation property of fuzzy complex numbers as follows:

Theorem 3.1 Let $\tilde{Z}_{k}=\tilde{X}_{k}+i \tilde{Y}_{k} \in F_{0}(C),(k=1,2)$, then for $\forall \alpha \in(0,1]$, we have
(1) $\left(\tilde{Z}_{1} \pm \tilde{Z}_{2}\right)_{\alpha}=Z_{1, \alpha} \pm Z_{2, \alpha}$.
(2) $\left(\tilde{Z}_{1} \cdot \tilde{Z}_{2}\right)_{\alpha}=Z_{1, \alpha} \cdot Z_{2, \alpha}$
(3) $\left(\tilde{Z}_{1} \div \tilde{Z}_{2}\right)_{\alpha}=Z_{1, \alpha} \div Z_{2, \alpha}$.
(4) $\left(k \tilde{Z}_{1}\right)_{\alpha}=k Z_{1, \alpha}, \quad k \in R$.

\section{The convergence of fuzzy complex number sequence}

Let $P\left(R^{n}\right)$ be the set of all non-empty compact convex subset on $R^{n}$. If $A_{1}, A_{2} \in P\left(R^{n}\right)$. Then Hausdorff distance is defined by

$$
H\left(A_{1}, A_{2}\right)=\inf \left\{\varepsilon>0 \mid A_{2} \subseteq B\left(A_{1}, \varepsilon\right), A_{1} \subseteq B\left(A_{2}, \varepsilon\right)\right\} \text { on } P\left(R^{n}\right)
$$

where $B(A, \varepsilon)=\left\{x \in R^{n} \mid d(x, A)<\varepsilon\right\}$ is a $\varepsilon$-neighborhood of a compact convex set $A, d(x, A)=\inf _{a \in A}\{|x-a|: a \in A\}$ is a distance from $x$ to a compact convex set $A$, where $|\cdot|$ is Euclid norm on $R^{n}$. 
We give uniform Hausdorff distance $D$ in $F_{0}(C)$, where $D$ is defined by

$$
D(\tilde{Z}, \tilde{W})=\sup _{0 \leq r \leq 1} H\left(\tilde{Z}_{r}, \tilde{W}_{r}\right), \forall \tilde{Z}, \tilde{W} \in F_{0}(C)
$$

Then $D$ is a perfect and non-separable distance on $F_{0}(C)$, and then $\left(F_{0}(c), D\right)$ is a perfect metric space. Since linear operation is continuous on $F_{0}(C)$, therefore $\left(F_{0}(C), D\right)$ is also a linear metric space. If we showed topological $T$ on $F_{0}(C)$, then $\left(F_{0}(C), T\right)$ is a topological space. Thus $\left(F_{0}(C), D\right)$ is a linear topological space too, denoted by $\left(F_{0}(C), D, T\right)$.

Definition 4.1 D-convergent: Let $\left\{\tilde{Z}_{0}, \tilde{Z}_{n}, n \geq 1\right\} \subseteq F_{0}(C),\left\{\tilde{Z}_{n}\right\}$ is said to converge to $\tilde{Z}_{0}$ in metric $D$ on $F_{0}(C)$, if and only if for $\forall \varepsilon>0, \exists N \in N^{+}$, such that $n>N$, we have

$$
D\left(\tilde{Z}_{n}, \tilde{Z}_{0}\right)<\varepsilon
$$

where $N^{+}=\{1,2, \cdots\}$. Denoted by $\tilde{Z}_{n} \stackrel{D}{\longrightarrow} \tilde{Z}_{0}$.

Definition 4.2 L-convergent: Let $\left\{\tilde{Z}_{0}, \tilde{Z}_{n}, n \geq 1\right\} \subseteq F_{0}(C),\left\{\tilde{Z}_{n}\right\}$ is said to converge to $\tilde{Z}_{0}$ in $r$-level on $F_{0}(C)$, if and only if for $\forall \varepsilon>0, \exists N \in N^{+}$, such that for $\forall 0 \leq r \leq 1, n>N$, we have

$$
H\left(\tilde{Z}_{n}^{r}, \tilde{Z}_{0}^{r}\right)<\varepsilon
$$

Denoted by $\tilde{Z}_{n} \underset{r}{\stackrel{L}{\longrightarrow}} \tilde{Z}_{0}$, where $\tilde{Z}_{n}^{r}, \tilde{Z}_{0}^{r}$ are $r$-cut level sets of $\tilde{Z}_{n}$ and $\tilde{Z}_{0}$ respectively .

In accordance with the definition of $D$-convergent and $L$-convergent, we have the following theorem:

Theorem 4.1 For $\left\{\tilde{Z}_{0}, \tilde{Z}_{n}, n \geq 1\right\} \subseteq F_{0}(C)$, we have $\tilde{Z}_{n} \stackrel{D}{\rightarrow} \tilde{Z}_{0} \Rightarrow \tilde{Z}_{n} \stackrel{L}{\rightarrow}$ $\tilde{Z}_{0}$.

Proof: Obvious .

\section{$5 \quad$ Fuzzy complex number-valued mapping and it's the fixed points}

Definition 5.1 Fuzzy complex number-valued mapping $f: F_{\tilde{\sim}}(C) \rightarrow F_{0}(C)$ is increasing, if for $\forall \tilde{Z}_{1}, \tilde{Z}_{2} \in F_{0}(C), \tilde{Z}_{1} \subseteq \tilde{Z}_{2}$, then $f\left(\tilde{Z}_{1}\right) \subseteq f\left(\tilde{Z}_{2}\right)$.

Definition 5.2 Let $\left(F_{0}(C), D\right)$ is a perfect metric space, $f: F_{0}(C) \rightarrow$ $F_{0}(C)$ is a fuzzy complex number-valued mapping, and $\tilde{Z}_{0} \in F_{0}(C)$

(1) $f$ is called continuous at $\tilde{Z}_{0}$, if and only if for $\forall \varepsilon>0, \exists \delta>0$, such that for $\forall \tilde{Z} \in F_{0}(C), D\left(\tilde{Z}, \tilde{Z}_{0}\right)<\delta$, we have

$$
D\left(f(\tilde{Z}), f\left(\tilde{Z}_{0}\right)\right)<\varepsilon
$$


(2) $f$ is called $r$-level convergent at $\tilde{Z}_{0}$, if and only if for $\forall \varepsilon>0, \exists \delta>0$, such that for $\forall \tilde{Z} \in F_{0}(C)$ and for $\forall r \in[0,1], H\left(\tilde{Z}^{r}, \tilde{Z}_{0}^{r}\right)<\delta$, we have

$$
H\left([f(\tilde{Z})]^{r},\left[f\left(\tilde{Z}_{0}\right)\right]^{r}\right)<\varepsilon
$$

Definition 5.3 Fuzzy complex number-valued mapping $f: F_{0}(C) \rightarrow$ $F_{0}(C)$ is called Lipschtz continuous, if for $\tilde{Z}, \tilde{Z}_{0} \in F_{0}(C)$, there exists a constant $L>0$, such that

$$
D\left[f(\tilde{Z}), f\left(\tilde{Z}_{0}\right)\right] \leq L \cdot D\left(\tilde{Z}, \tilde{Z}_{0}\right)
$$

where $L$ is a Lipschtz constant.

Definition 5.4 Let $\left(F_{0}(C), D\right)$ is a metric space, $f: F_{0}(C) \rightarrow F_{0}(C)$ is a fuzzy complex number-valued mapping, if $\exists \alpha \in(0,1)$, such that

$$
\forall \tilde{Z}, \tilde{W} \in F_{0}(C), D(f(\tilde{Z}), f(\tilde{W})) \leq \alpha \cdot D(\tilde{Z}, \tilde{W})
$$

then $f$ is called a fuzzy complex number-valued compression mapping on $F_{0}(C)$.

Obviously, a fuzzy complex number-valued compression mapping must be a continuous mapping on $F_{0}(C)$. It can be proved directly by using the definition above.

\section{Main Results}

Theorem 5.1 Let $\left(F_{0}(C), D\right)$ is a perfect metric space, $f$ is a fuzzy complex number-valued compression mapping on $\left(F_{0}(C)\right.$, then $f$ exists a unique fixed point on $\left(F_{0}(C)\right.$.

Proof: For $\forall \tilde{Z}_{0} \in F_{0}(C)$ let

$$
\tilde{Z}_{1}=f\left(\tilde{Z}_{0}\right), \tilde{Z}_{2}=f\left(\tilde{Z}_{1}\right)=f^{2}\left(\tilde{Z}_{0}\right), \cdots, \tilde{Z}_{n}=f\left(\tilde{Z}_{n-1}\right)=f^{n}\left(\tilde{Z}_{0}\right), \cdots
$$

In the following we prove that $\left\{\tilde{Z}_{n}\right\}$ is $D$-convergent on $F_{0}(C)$.

In fact,

$$
\begin{aligned}
D\left(\tilde{Z}_{m+1}, \tilde{Z}_{m}\right) & =D\left(f\left(\tilde{Z}_{m}\right), f\left(\tilde{Z}_{m-1}\right)\right) \leq \alpha \cdot D\left(\tilde{Z}_{m}, \tilde{Z}_{m-1}\right) \\
& =\alpha \cdot D\left(f\left(\tilde{Z}_{m-1}\right), f\left(\tilde{Z}_{m-2}\right)\right) \leq \alpha^{2} \cdot D\left(\tilde{Z}_{m-1}, \tilde{Z}_{m-2}\right) \\
& \leq \cdots \leq \alpha^{m} \cdot D\left(\tilde{Z}_{1}, \tilde{Z}_{0}\right)
\end{aligned}
$$

thus when $n>m$, we have

$$
\begin{aligned}
D\left(\tilde{Z}_{m}, \tilde{Z}_{n}\right) & \leq D\left(\tilde{Z}_{m}, \tilde{Z}_{m+1}\right)+\cdots+D\left(\tilde{Z}_{n-1}, \tilde{Z}_{n}\right) \\
& \leq\left(\alpha^{m}+\alpha^{m-1}+\cdots+\alpha^{n-1}\right) D\left(\tilde{Z}_{0}, \tilde{Z}_{1}\right) \\
& =\alpha^{m} \frac{1-\alpha^{n-m}}{1-\alpha} D\left(\tilde{Z}_{0}, \tilde{Z}_{1}\right)
\end{aligned}
$$


Since $0<\alpha<1$ so when $n>m$, we have

$$
D\left(\tilde{Z}_{m}, \tilde{Z}_{n}\right) \leq \alpha^{m} \frac{1}{1-\alpha} D\left(\tilde{Z}_{0}, \tilde{Z}_{1}\right) \rightarrow 0 \quad(m \rightarrow \infty)
$$

Therefore $\left\{\tilde{Z}_{n}\right\}$ is $D$-convergent on $F_{0}(C)$. Since

By the perfection of $F_{0}(C)$, then $\exists \tilde{Z} \in F_{0}(C)$ such that $\tilde{Z}_{n} \rightarrow \tilde{Z}(n \rightarrow \infty)$.

$$
\begin{aligned}
D(\tilde{Z}, f(\tilde{Z})) & \leq D\left(\tilde{Z}, \tilde{Z}_{m}\right)+D\left(\tilde{Z}_{m}, f(\tilde{Z})\right) \\
& \leq D\left(\tilde{Z}, \tilde{Z}_{m}\right)+\alpha \cdot D\left(\tilde{Z}_{m-1}, \tilde{Z}\right)
\end{aligned}
$$

Hence when $m \rightarrow \infty$, we have $D(\tilde{Z}, f(\tilde{Z})) \rightarrow 0$, therefore $D(\tilde{Z}, f(\tilde{Z}))=0$, i.e. $f(\tilde{Z})=\tilde{Z}$.

Uniqueness : if there exists another $\tilde{Z}^{\prime} \in F_{0}(C)$, such that $f\left(\tilde{Z}^{\prime}\right)=\tilde{Z}^{\prime}$, then

$$
D\left(\tilde{Z}, \tilde{Z}^{\prime}\right)=D\left(f(\tilde{Z}), f\left(\tilde{Z}^{\prime}\right)\right) \leq \alpha \cdot D\left(\tilde{Z}, \tilde{Z}^{\prime}\right)
$$

Because $0<\alpha<1$, thus $D\left(\tilde{Z}, \tilde{Z}^{\prime}\right)=0$, i.e. $\tilde{Z}=\tilde{Z}^{\prime}$.

This complete the proof of the theorem.

Let $\tilde{Z}_{0} \in F_{0}(C)$ is a fuzzy complex number, denotes the $\varepsilon$-spheroid (hyper spheroid) of $\tilde{Z}_{0}$ by $B\left(\tilde{Z}_{0}, \varepsilon\right)=\left\{\tilde{Z} \in F_{0}(C) \mid D\left(\tilde{Z}, \tilde{Z}_{0}\right)<\varepsilon\right\}, \bar{B}\left(\tilde{Z}_{0}, \varepsilon\right)$ is the $\varepsilon$-closed spheroid of $\tilde{Z}_{0}$. So we have the following theorem that the result is much better than in Theorem 5.1

Theorem 5.2 Let $\left(F_{0}(C), D\right)$ is a perfect metric space, $f: F_{0}(C) \rightarrow F_{0}(C)$ is a compression mapping on $\bar{B}\left(\tilde{Z}_{0}, \varepsilon\right)$, and $D\left(f\left(\tilde{Z}_{0}\right), \tilde{Z}_{0}\right) \leq(1-\alpha) \varepsilon, \alpha \in[0,1)$. Then $f$ has a unique fixed point on $\bar{B}\left(\tilde{Z}_{0}, \varepsilon\right)$.

Proof: we can prove: For $\forall \tilde{Z} \in \bar{B}\left(\tilde{Z}_{0}, \varepsilon\right)$, then $f(\tilde{Z}) \in \bar{B}\left(\tilde{Z}_{0}, \varepsilon\right)$. In fact for $\forall \tilde{Z} \in \bar{B}\left(\tilde{Z}_{0}, \varepsilon\right)$, i.e. $D\left(\tilde{Z}, \tilde{Z}_{0}\right) \leq \varepsilon$, Then

$$
\begin{aligned}
D\left(f(\tilde{Z}), \tilde{Z}_{0}\right) & \leq D\left(f(\tilde{Z}), f\left(\tilde{Z}_{0}\right)\right)+D\left(f\left(\tilde{Z}_{0}\right), \tilde{Z}_{0}\right) \\
& \leq \alpha \cdot D\left(\tilde{Z}, \tilde{Z}_{0}\right)+(1-\alpha) \varepsilon \\
& \leq \alpha \varepsilon+(1-\alpha) \varepsilon=\varepsilon
\end{aligned}
$$

i.e. $f(\tilde{Z}) \in \bar{B}\left(\tilde{Z}_{0}, \varepsilon\right)$.

Since closed spheroid $\bar{B}\left(\tilde{Z}_{0}, \varepsilon\right)$ is a closed set of the perfect metric space, and it is a perfect metric space too. Therefore Theorem 5.2 is correct by Theorem 5.1.

Theorem 5.3 Let $\left(F_{0}(C), D\right)$ is a perfect metric space, $\tilde{W}=g(\tilde{Z})$ is a fuzzy complex number-valued mapping on $F_{0}(C)$. If $\exists n \in N^{+}$such that $g^{n}$ is a compression mapping on $F_{0}(C)$, then $g$ exists a unique fixed point on $F_{0}(C)$.

Proof: Let $f=g^{n}$, then $f$ is a compression mapping on $F_{0}(C)$, then by Theorem 5.1. $f$ exists a unique fixed point $\tilde{Z}_{0}$ on $F_{0}(C)$. i.e. $\tilde{Z}_{0}=f\left(\tilde{Z}_{0}\right)$. 
In the following, we prove that $\tilde{Z}_{0}$ is the fixed point of $g$. In fact, since $f g=g^{n}=g f$, thus $f\left(g\left(\tilde{Z}_{0}\right)\right)=g\left(f\left(\tilde{Z}_{0}\right)\right)=g\left(\tilde{Z}_{0}\right)$.

Therefore $g\left(\tilde{Z}_{0}\right)$ is the fixed point of $f$ too, because the fixed point of $f$ is unique, so that $g\left(\tilde{Z}_{0}\right)=\tilde{Z}_{0}$.

Uniqueness: Suppose $\tilde{Z}_{1}$ is an arbitrary fixed point of $g$, since $g\left(\tilde{Z}_{1}\right)=\tilde{Z}_{1}$ we have

$$
g^{n}\left(\tilde{Z}_{1}\right)=g^{n-}\left(\tilde{Z}_{1}\right)=\cdots=\tilde{Z}_{1}
$$

Hence $\tilde{Z}_{1}$ is also a fixed point of $f=g^{n}$, because $f$ exists only one fixed point, thus we have $\tilde{Z}_{1}=\tilde{Z}_{0}$.

Therefore, $f=g^{n}$ exists a unique fixed point $\tilde{Z}_{0}$ on $F_{0}(C)$.

Note: when $n=1$, then Theorem 5.2 becomes Theorem 5.1. that is to say Theorem 5.1 is the especial case of Theorem 5.2.

Theorem 5.4 Let $\left(F_{0}(C), D\right)$ is a perfect metric space, $f: F_{0}(C) \rightarrow F_{0}(C)$, $a_{n}=\sup _{\tilde{Z}, \tilde{W} \in F_{0}(C)} \frac{D\left(f^{n}(\tilde{Z}), f^{n}(\tilde{W})\right)}{D(\tilde{Z}, \tilde{W})} \rightarrow 0,(n \rightarrow \infty)$, then $f$ exists a unique fixed point on $F_{0}(C)$.

Proof: Since $\lim _{n \rightarrow \infty} a_{n}=0$, so that when $n>N$, we have $a_{n} \leq \frac{1}{2}$. Thus for $\forall \tilde{Z}, \tilde{W} \in F_{0}(C), \frac{D\left(f^{n}(\tilde{Z}), f^{n}(\tilde{W})\right)}{D(\tilde{Z}, \tilde{W})} \leq \frac{1}{2}$ i.e. $D\left(f^{n}(\tilde{Z}), f^{n}(\tilde{W})\right) \leq \frac{1}{2} D(\tilde{Z}, \tilde{W})$. Then by the Theorem 5.3, $f$ exists a unique fixed point on $F_{0}(C)$.

Theorem 5.5 Let $B$ be a bounded closed set on $F_{0}(C), f: B \rightarrow B$, For $\forall \tilde{Z}, \tilde{W} \in F_{0}(C)$, when $\tilde{Z} \neq \tilde{W}$ we have $D(f(\tilde{Z}), f(\tilde{W}))<D(\tilde{Z}, \tilde{W})$ then $f$ exists a unique fixed point on $B$.

Proof: we prove $f$ is a compression mapping first. i.e. $\exists \alpha \in(0,1]$, such that for $\forall \tilde{Z}, \tilde{W} \in B$ we have

$$
D(f(\tilde{Z}), f(\tilde{W})) \leq \alpha D(\tilde{Z}, \tilde{W})
$$

Suppose it is not true, then for $\forall n$ we have $\tilde{Z}_{n}, \tilde{W}_{n} \in B$, such that

$$
D\left(f\left(\tilde{Z}_{n}\right), f\left(\tilde{W}_{n}\right)\right)>\left(1-\frac{1}{n}\right) D\left(\tilde{Z}_{n}, \tilde{W}_{n}\right)
$$

Since $B$ is a bounded closed set on $F_{0}(C)$ then $B$ is a compact set, therefore there exists $\left\{\tilde{Z}_{n_{k}}\right\},\left\{\tilde{W}_{n_{k}}\right\}$ and $\tilde{Z}_{0}, \tilde{W}_{0} \in B$, such that $\tilde{Z}_{n_{k}} \rightarrow \tilde{Z}_{0}, \tilde{W}_{n_{k}} \rightarrow \tilde{W}_{0}$. In (4), we substitute $\tilde{Z}_{n}, \tilde{W}_{n}$ with $\tilde{Z}_{n_{k}}, \tilde{W}_{n_{k}}$ respectively, and then let $k \rightarrow \infty$, we have

$$
\begin{aligned}
D\left(f\left(\tilde{Z}_{0}\right), f\left(\tilde{W}_{0}\right)\right) & =\lim _{k \rightarrow \infty} D\left(f\left(\tilde{Z}_{n_{k}}\right), f\left(\tilde{W}_{n_{k}}\right)\right) \\
& \geq \lim _{k \rightarrow \infty}\left(1-\frac{1}{n}\right) D\left(\tilde{Z}_{n_{k}}, \tilde{W}_{n_{k}}\right) \\
& =D\left(\tilde{Z}_{0}, \tilde{W}_{0}\right)
\end{aligned}
$$

This contradicts with the supposition of the theorem, therefore (3) is correct.

By the Theorem 5.3, $f$ exists a unique fixed point on $B$. 


\section{References}

[1] J.J.Buckley. Fuzzy complex numbers. Fuzzy Sets and Systems, 33(1989)333-345.

[2] J.J.Buckley. Fuzzy complex analysis I: Differentiation, Fuzzy Sets and Systems.(1991)41:269-284.

[3] J.J.Buckley. Fuzzy complex analysis II: Integration, Fuzzy Sets and Systems (1992)49:171-179.

[4] Ma Shengquan, Fuzzy Complex Numbers and Some Operational Properties, Journalof Lanzhou University, (Natural Science Edition), (1996)32, 643-645.

[5] Ma Shengquan and Cao Chun, The Differentiation of Complex Fuzzy Functions, Proceeding of the 9-th National Conference of Fuzzy Mathematics and Fuzzy Systems, Baoding, Hebei University Press (1998).162-166.

[6] Ma Shengquan and Cao Chun, Fuzzy Complex Analysis, The Ethnic House ,Beijing(2001).

[7] Ma Shengquan and Ji Jinshui, Uniform convergency in the series of complex interval-valued function and complex fuzzy-valued function, Journal of Liaoning Technical University, (Natural Science Edition), 20(2001), 615617.

[8] Ma Shengquan, A Further Discussion on the Series of Complex Fuzzy Valued and its Convergence, Fuzzy Systems and Mathematics(Chinese), Vo1.16.No.2.(2002):92-96.

[9] Ma shengquan and Gong zengtai, The Series of Complex Fuzzy Number and Complex Fuzzy-valued Functions and Its Convergence, International Journal of Pure and Applied Mathematics. Vol.7.No.1.2003.4962.(Bulgaria).

[10] Ma shengquan, The Series of Complex fuzzy Valued and its Convergence, The Journal of Fuzzy Mathematics, Vol.13No.2(2005)(USA)467-475.

[11] Ma Shengquan and Gong Zengtai, The Rsearch Advances in Fuzzy Complex Analysis, Mathematics in Practice and Theory(Chinese), (2006)Vol.36.No.5:200-211.

\section{Received: October 25, 2006}

